\title{
Associação entre variáveis psicológicas e asma: uma revisão de literatura
}

The association between psychological variables and asthma: a literature review

Asociación entre variables psicológicas y asma: una revisión de la literatura

Naiara França Silva*

\section{Resumo}

Esta pesquisa objetivou levantar os principais achados acerca da relação entre a asma em crianças e adolescentes e variáveis psicossociais e ou psicológicas, publicados em periódicos nacionais nos últimos 15 anos. Usou-se o método de revisão de literatura, enfocando estudos voltados para os aspectos psicológicos e sociais presentes no desencadeamento e ou agravamento da asma, considerando o paciente e a sintomatologia asmática como foco de investigação. Constatou-se que, de modo geral, os resultados confirmam a associação entre as variáveis psicológicas, desencadeamento de crises e agravamento de doença em indivíduos asmáticos. Tais resultados sugerem a necessidade de que novas propostas terapêuticas englobem o envolvimento de equipes multiprofissionais e os estudos reforçaram a importância de uma abordagem sistêmica do paciente asmático e seus familiares. Pesquisas futuras, com amostras maiores e com diferentes grupos de crianças com doenças crônicas, poderiam auxiliar a compreender melhor o impacto psicossocial destas sobre o desenvolvimento do indivíduo.

Palavras-chave: Asma, Variáveis psicossociais, Abordagem sistêmica.

\begin{abstract}
This research is aimed at assessing the main findings concerning the relationship between asthma in children and adolescents and psychosocial and/or psychological variables, which were published in national journals in the last fifteen years. We used the method of literature review, focusing on studies into the psychological and social aspects present in triggering and/or worsening of asthma, whereas asthma symptoms and the patient as a focus of investigation. It was found that overall, the results confirm the association between psychological variables, triggering crisis and worsening of disease in individuals with asthma. These results suggest the need for new therapeutic approaches that encompass the involvement
\end{abstract}

Mestranda em Psicologia Social pela Universidade Federal de Sergipe (UFS), psicóloga. E-mail: naiarafs_psico@yahoo. com.br. 
of multidisciplinary teams and studies reinforced the importance of a systems approach to asthma patients and their families. Future research with larger samples and with different groups of children with chronic diseases, could help to better understand the psychosocial impact of these on the development of the individual.

Keywords: Asthma, Psychosocial variables, Systemic approach.

\section{Resumen}

Esta investigación tuvo como objetivo plantear las principales conclusiones acerca de la relación entre el asma en niños y adolescentes y las variables psicosociales y/o psicológicas publicadas en revistas nacionales en los últimos 15 años. Se utilizó el método de revisión de la literatura, centrándose en los estudios dirigidos a los aspectos psicológicos y sociales presentes en el desencadenamiento o empeoramiento del asma y considerando al paciente y la sintomatología asmática como foco de la investigación. Se constató que, en general, los resultados confirman la asociación entre las variables psicológicas, surgimiento de crisis y empeoramiento de la enfermedad en pacientes asmáticos. Estos resultados sugieren la necesidad de que nuevos enfoques terapéuticos abarquen la participación de equipos multidisciplinarios, y los estudios reforzaron la importancia de un enfoque sistémico de los pacientes asmáticos y de sus familias. Estudios futuros, con muestras de mayor tamaño y con diferentes grupos de niños con enfermedades crónicas, podrían ayudar a comprender mejor el impacto psicosocial de dichas enfermedades en el desarrollo del individuo.

Palabras clave: Asma, Variables psicosociales, Enfoque sistémico.

asma é uma doença crônica com diversas causas, sintomas e consequências
para a saúde do doente. Atualmente essa doença representa um problema
social de magnitude considerável, tornando-se relevante conhecêla melhor por meio de estudos que venham a contribuir para uma efetiva melhoria da qualidade de vida dos asmáticos. Sobre a asma ativa, caracterizada pela constância de sintomas nos últimos 12 meses, Nogueira, Silva e Lopes (2009) citaram que há variabilidade na sua prevalência no mundo, variando de 1,6 a 36,8\%, estando o Brasil em $8^{\circ}$ lugar, com a prevalência média de $20 \%$. Qualquer que seja a gravidade da asma, ocorre redução nos domínios físico, psicológico e social (Mello Filho, 2002; Nogueira, 2007). 
A existência de uma doença crônica, a exemplo da asma, envolve um complexo processo de adaptação. Essa situação traz consigo um conjunto de alterações que podem desafiar a visão que o indivíduo tem de si, das suas capacidades e do mundo, o que inclui alteraçôes de papéis sociais e profissionais, autocuidado, sofrimento físico e psicológico, por vezes, complexas e prolongadas (Matos \& Machado, 2007). Em crianças e adolescentes, a presença de uma doença crônica pode afetar negativamente o desenvolvimento, por restringir a realização de atividades típicas da faixa etária, dificultar a socialização e o desenvolvimento da autoimagem positiva, além de aumentar a vulnerabilidade para transtornos comportamentais (Salomão Júnior, Miyazaki, Cordeiro, Domingos \& Valério, 2008).

Várias pesquisas mostraram também a relevância de fatores psicológicos interferindo no manejo da asma. Ansiedade, depressão, negação da doença e presença de conflitos familiares têm sido associados à menor adesão ao tratamento e maior morbimortalidade (Campanha, Freire \& Fontes, 2008). A maioria dos estudos sobre a associação de transtornos emocionais e comportamentais e asma realizados em crianças e adolescentes mostra que eles têm maior prevalência de transtornos emocionais do que a população geral (Alvim, Ricas, Camargos, Lasmar, Andrade et al., 2008; Campanha, Freire \& Fontes, 2008; Matos \& Machado, 2008; Nogueira, Silva \& Lopes, 2009; Salomão Júnior et. al. 2008).

De acordo com Borba, Ribeiro, Ohara e Sarti (2009), a asma envolve não só o aspecto biológico, mas também as relações interpessoais em seus aspectos psicológicos e sociais, transformando-se em experiências difíceis, permeadas de sofrimento. Desse modo, entende-se que a participação de um componente emocional ou psicodinâmico no quadro da asma brônquica é um fenômeno marcante e bem conhecido. Tal componente emocional pode influir em três níveis do quadro asmático: no desencadeamento das crises, na persistência ou agravamento do sofrimento durante as crises ou períodos intercríticos e na resistência do tratamento (Lemle, 2010).

O componente psicológico dessas crises deve ser bem estudado pelos profissionais de saúde que interagem com pessoas com asma, objetivando o combate à ansiedade e ao sofrimento causado pelas limitações vivenciadas. Além da ansiedade decorrente da doença, aquela pode estar vinculada a outros fatores, que podem contribuir para o desencadeamento de crises, tais como um atendimento clínico insatisfatório, conflitos familiares e 
falta de conhecimento sobre a asma. Portanto cabe ao profissional estar atento para razões psicológicos e psicossociais por parte do asmático, principalmente quando este estiver passando por longo período de sintomas ou crises repetidas. Como foi assegurado por Lemle (2010), os fatores psicológicos atuam na intercrise com consequências por vezes muito fortes, mantendo ou agravando o sofrimento e podendo conduzir à resistência ao tratamento.

Embora nem sempre sejam concordantes os resultados encontrados pelas diferentes investigações, torna-se cada vez mais evidente que os fatores psicossociais desempenham um papel importante na asma brônquica, quer como elemento precipitante das crises, quer na progressão da doença. Nesse cerne, concentra-se a importância de conhecer as principais contribuiçôes de estudos existentes no Brasil a respeito de fatores psicológicos e sociais influentes no adoecimento/crise da asma, a fim de conduzir a uma abordagem sistêmica e interdisciplinar no tratamento de pacientes asmáticos.

No Brasil, pesquisas vêm sendo desenvolvidas com o objetivo de relacionar a asma a fatores psicológicos e psicossociais. No entanto não há uma síntese desses achados, como também não se sabe de que modo estão apresentadas as principais questóes abordadas na literatura nacional. Sendo assim, percebe-se a existência de uma lacuna no que tange ao estado atual do conhecimento acerca da asma no âmbito dos estudos brasileiros, tornando-se pertinente, portanto, mapear as principais contribuições e investigações conduzidas dentro dessa temática.

Logo, o objetivo deste estudo é levantar os principais achados acerca da relação entre a asma em crianças e adolescentes, e variáveis psicossociais e ou psicológicas, publicados em estudos nacionais da Psicologia e ciências afins, indexados em algumas bases de dados eletrônicas brasileiras, nos últimos 15 anos. Além disso, pretende-se sistematizar as principais propostas executadas no que se refere a objetivos, método e abordagem de análise, revelando os mais comuns desenhos de estudo, questionamentos e conclusões apresentados pelos pesquisadores nesse campo de investigações.

\section{Método}

Empregou-se o método de revisão de literatura, realizando-se pesquisa em importantes bases de dados científicos brasileiros, disponíveis por via eletrônica e de acesso livre - Scielo (www.scielo.br) e Pepsic (pepsic. 
bvsalud.org) - e nos bancos de dados de teses e dissertaçôes, nas quais se buscaram trabalhos publicados até setembro de 2010 , por meio da palavrachave "asma”. Enfocaram-se todos os estudos resultantes do levantamento inicial, desde que fossem voltados para os aspectos psicológicos e sociais presentes no desencadeamento e ou agravamento da asma em crianças e adolescentes, considerando o paciente e a sintomatologia asmática como foco de investigação. Para tanto, os critérios de exclusão foram: não ter sido realizado no Brasil, privilegiar o enfrentamento da equipe médica e não os pacientes, e envolver pacientes com outras doenças crônicas.

De acordo com os parâmetros mensurados, os trabalhos foram subdivididos em três grupos: a) relação entre construtos psicológicos e a asma; b) influência de fatores sociais e ou psicossociais na doença; e c) influência de fatores biopsicossociais na asma.

Consideraram-se construtos psicológicos os aspectos emocionais que interferem na doença. A respeito dos fatores sociais e ou psicossociais, aglutinaram-se os estudos que visavam a investigar a influência destes na ocorrência da asma. Por fim, assinalaram-se como variáveis biopsicossociais aqueles estudos que buscaram englobar o contexto integral do paciente, caracterizando-se como uma dimensão mais interativa e complexa da funcionalidade entre dinâmicas psicológicas, biológicas e sociais, todas mutuamente influentes no adoecimento e ou manutenção da asma.

\section{Resultados e discussão}

Foram localizados, ao todo, 417 estudos ao se usar o termo "asma" nas bases de dados. Após a leitura dos resumos e eliminação de títulos incompatíveis com esta revisão, restaram 63 artigos, observando-se os critérios de inclusão e exclusão. Os 63 artigos pré-selecionados foram minuciosamente reavaliados quanto aos critérios estabelecidos (de inclusão e exclusão), sendo então excluídos 24, restando 38 a serem captados para esta revisão.

Para a exploração dos resultados e a discussão, inicialmente foi feito um apanhado dos estudos que buscaram analisar os aspectos psicológicos como fatores desencadeantes da asma, sendo, em seguida, levantados os trabalhos que enfocaram os aspectos psicológicos como agravantes da doença. No terceiro tópico, foram trazidos estudos voltados para a influência de fatores psicossociais nos asmáticos, sendo, por fim, apresentadas pesquisas que enfatizaram as variáveis biopsicossociais, conforme tabela 1 . 
Tabela 1 - Levantamento de publicações sobre asma encontradas nas bases de dados Scielo e Pepsic, no período entre junho e setembro de 2010, com a palavra-chave "asma".

\begin{tabular}{|c|c|c|c|}
\hline Autor/ano & & Amostra & $\begin{array}{l}\text { Método } \\
\text { Instrumentos e delineamentos }\end{array}$ \\
\hline $\begin{array}{l}\text { Rockenbach } \\
\text { (1993) }\end{array}$ & $\begin{array}{l}\text { Padrões de interação } \\
\text { mãe-criança em díades } \\
\text { com crianças asmáticas }\end{array}$ & 37 díades & $\begin{array}{l}\text { O desenvolvimento físico, psico- } \\
\text { motor e emocional das crianças } \\
\text { foi avaliado por meio de uma en- } \\
\text { trevista com as mães. A interação } \\
\text { mãe-criança e criança-estranha } \\
\text { foi examinada por uma sessão de } \\
\text { observações que foi filmada para } \\
\text { posterior análise. }\end{array}$ \\
\hline $\begin{array}{l}\text { Peçanha } \\
\text { (1997) }\end{array}$ & $\begin{array}{l}\text { A reciprocidade de } \\
\text { desenvolvimento entre } \\
\text { a criança com asma e } \\
\text { sua família }\end{array}$ & 10 sujeitos & $\begin{array}{l}\text { Pesquisa comparativa. O exame } \\
\text { psicológico compreendeu: a) } \\
\text { entrevista com os pais; b) teste } \\
\text { do desenho colorido da família; c) } \\
\text { teste das fábulas com as crianças; } \\
\text { d) entrevista estruturada familiar. }\end{array}$ \\
\hline $\begin{array}{l}\text { Rodrigues } \\
\text { (1997) }\end{array}$ & $\begin{array}{l}\text { Contribuições de } \\
\text { técnicas de exame psi- } \\
\text { cológico com crianças } \\
\text { asmáticas e familiares }\end{array}$ & 20 sujeitos & $\begin{array}{l}\text { Os instrumentos de avaliação } \\
\text { usados foram: teste de apercep- } \\
\text { ção temática infantil (CAT-A) e o } \\
\text { desenho da figura humana (DHF) } \\
\text { nas crianças; teste de apercepção } \\
\text { temática (TAT); e entrevista semi- } \\
\text { dirigida nas mães. }\end{array}$ \\
\hline
\end{tabular}

Estudo da relação entre

Rahim (2000) asma infantil e privação psicológica
50 sujeitos

Aplicou-se entrevista dirigida com as mães.
Um estudo psicológico

da asma em criança:

Buzinari considerações acerca

(2001) da angústia e inibição

presente nessa sinto-

matologia
12 díades

Competência Social e

Salomão

Júnior (2001)

62 sujeitos transtornos compor-

tamentais em crianças

portadores de asma

moderada e grave
Pesquisa na teoria freudiana e um estudo de casos, realizado por meio de entrevistas semiestruturadas e análises pela escuta.

Foram utilizados na obtenção dos dados uma ficha de identificação, uma ficha de dados clínicos e um instrumento que avalia competência social e transtornos comportamentais - child behavior checklist (CBCL). 


\section{Autor/ano}

\section{Amostra Método \\ Instrumentos e delineamentos}

Aspectos emocionais,

Santos (2001) físicos e ambientais associados ao quadro de asma brônquica
Estudo descritivo realizado por meio de questionário sob forma de entrevista semidirigida e dois instrumentos psicológicos para avaliação do grau de ansiedade e depressão.
Função materna e os

Oliveira (2002) fenômenos psicossomáticos: reflexões a 5 díades Estudo de caso partir da asma infantil

Atopia, fumo passivo, infecções respiratórias

Ribeiro et al. (2002) e asma entre crianças do jardim de infância e da escola primária
Estudo descritivo. Foi administrado um questionário contendo 31 questões aos responsáveis pelas 183 sujeitos crianças e realizados 88 testes cutâneos de alergia para inalantes nas crianças cujos pais haviam dado consentimento.

Estudo de caso de natureza qualitativa. As técnicas de coleta de dados foram a observação par-

Borba e Sarti (2005)

A asma infantil e o mundo social e familiar da criança

3 sujeitos ticipante, a entrevista com roteiro prévio e o brinquedo terapêutico, que definiram os eixos em torno dos quais foi feita a análise.

Trata-se de um estudo transversal.

Depressão e transtornos psiquiátricos menores em mães de crianças com asma
Escalas BDI, versão brasileira de

240 sujeitos Andrade \& Gorestein e a escala SRQ-20, validada para o Brasil por Mari \& Willians.

$\begin{array}{ll}\text { Berenchtein } & \text { A influência do stress } \\ \text { (2004) } & \text { na expressão clínica da } \\ & \text { asma infantil }\end{array}$

Berenchtein $(2004)$
Utilizou-se a escala de stress infantil (Lipp \& Lucarelli, 1998) e um questionário aplicado aos pais.

$\begin{array}{ll} & \text { Alergias respiratórias } \\ \text { Castro (2004) } & \begin{array}{l}\text { e qualidade de vida: } \\ \text { interface medicina e } \\ \text { psicanálise }\end{array}\end{array}$

Aplicação do questionário de QV SF-36. Foi analisado um caso 100 sujeitos clínico por meio do referencial psicanalítico de orientação lacaniana. 


\begin{tabular}{|c|c|c|c|}
\hline Autor/ano & & Amostra & $\begin{array}{c}\text { Método } \\
\text { Instrumentos e delineamentos }\end{array}$ \\
\hline Haidar (2004) & $\begin{array}{l}\text { Aspectos clínicos, so- } \\
\text { cioeconômicos e labo- } \\
\text { ratoriais de crianças } \\
\text { asmáticas atendidas } \\
\text { em um hospital público } \\
\text { de São Luís-MA }\end{array}$ & 169 sujeitos & $\begin{array}{l}\text { Um questionário estruturado foi } \\
\text { aplicado às mães, e amostras de } \\
\text { sangue e fezes foram coletadas } \\
\text { para análises laboratoriais. }\end{array}$ \\
\hline Santos (2004) & $\begin{array}{l}\text { Asma infantil: refletin- } \\
\text { do o cuidado materno }\end{array}$ & 7 sujeitos & $\begin{array}{l}\text { Metodologia qualitativa, com es- } \\
\text { tudo etnográfico, dado o referen- } \\
\text { cial teórico proposto. }\end{array}$ \\
\hline $\begin{array}{l}\text { Lima, L. H. } \\
(2005)\end{array}$ & $\begin{array}{l}\text { Vivenciando o cuidar } \\
\text { do filho asmático: } \\
\text { respostas emocionais } \\
\text { da mãe }\end{array}$ & 9 sujeitos & $\begin{array}{l}\text { Pesquisa de natureza qualitativa e } \\
\text { descritiva, sendo realizada entrev- } \\
\text { ista com os sujeitos de estudo. }\end{array}$ \\
\hline $\begin{array}{l}\text { Lima, L. M. } \\
(2005)\end{array}$ & $\begin{array}{l}\text { A criança com asma: } \\
\text { estudo de adaptação } \\
\text { psicológica e de algu- } \\
\text { mas variáveis preditivas }\end{array}$ & 89 crianças & $\begin{array}{l}\text { Os instrumentos de avaliação } \\
\text { utilizados foram: school-age } \\
\text { temperament inventary (SATI), } \\
\text { teste das matrizes progressivas } \\
\text { coloridas de Raven, subescala de } \\
\text { avaliação da autoestima global de } \\
\text { Harter, schoolagen coping strate- } \\
\text { gies inventory de Ryan - Wenger } \\
\text { e um questionário construído } \\
\text { para estudo preenchido pela mãe } \\
\text { ou cuidador principal da criança. }\end{array}$ \\
\hline $\begin{array}{l}\text { Mendonça } \\
\text { \& Ferreira } \\
(2005)\end{array}$ & $\begin{array}{l}\text { Adesão ao tratamento } \\
\text { da asma na infância: } \\
\text { dificuldades enfrenta- } \\
\text { das por cuidadoras }\end{array}$ & 10 sujeitos & $\begin{array}{l}\text { Foram utilizados prontuários } \\
\text { médicos, um roteiro de entrevista } \\
\text { e a escala modos de enfrenta- } \\
\text { mento de problemas. }\end{array}$ \\
\hline $\begin{array}{l}\text { Baldessar } \\
(2006)\end{array}$ & $\begin{array}{l}\text { Fatores de risco para } \\
\text { asma e rinite em ado- } \\
\text { lescentes de criciúma }\end{array}$ & 73 sujeitos & $\begin{array}{l}\text { Todos responderam a um ques- } \\
\text { tionário sobre os fatores de risco } \\
\text { e foram realizadas dosagens de IgE } \\
\text { sérica, testes cutâneos de leitura } \\
\text { imediata, citologia nasal e espi- } \\
\text { rometria com broncodilatação; e } \\
\text { aqueles com critérios de asma e } \\
\text { espirometria normal realizaram } \\
\text { broncoprovocação com metaco- } \\
\text { lina. }\end{array}$ \\
\hline
\end{tabular}




\begin{tabular}{|c|c|c|c|}
\hline Autor/ano & & Amostra & $\begin{array}{c}\text { Método } \\
\text { Instrumentos e delineamentos }\end{array}$ \\
\hline $\begin{array}{l}\text { Fregonese } \\
\text { (2006) }\end{array}$ & $\begin{array}{l}\text { Estudo sobre o psico- } \\
\text { dinamismo de pais de } \\
\text { crianças atendidas em } \\
\text { programa de alegrias } \\
\text { respiratórias }\end{array}$ & ------ & $\begin{array}{l}\text { Foram usados como instrumentos } \\
\text { de investigação entrevistas semi- } \\
\text { dirigidas e duas pranchas do teste } \\
\text { de apercepção temática (TAT). }\end{array}$ \\
\hline $\begin{array}{l}\text { Resende } \\
(2006)\end{array}$ & $\begin{array}{l}\text { Identificação dos } \\
\text { fatores associados ao } \\
\text { atraso do desenvolvim- } \\
\text { ento motor em crianças } \\
\text { de um a quatro anos de } \\
\text { idade com asma }\end{array}$ & 99 sujeitos & $\begin{array}{l}\text { O desenvolvimento motor das } \\
\text { crianças foi avaliado com o teste } \\
\text { pediatric evaluation of disabil- } \\
\text { ity inventory. Um questionário de } \\
\text { saúde foi aplicado para verificar } \\
\text { fatores biológicos relacionados ao } \\
\text { atraso do desenvolvimento motor. }\end{array}$ \\
\hline $\begin{array}{l}\text { Valença et al. } \\
\text { (2006) }\end{array}$ & $\begin{array}{l}\text { Relação entre a } \\
\text { gravidade da asma e } \\
\text { comorbidade com tran- } \\
\text { stornos de ansiedade e } \\
\text { depressão }\end{array}$ & 62 sujeitos & $\begin{array}{l}\text { Entrevista com a utilização do } \\
\text { Mini-International Neuropsychiat- } \\
\text { ric Interview } 4.4 \text { Version. }\end{array}$ \\
\hline Aragão (2007) & $\begin{array}{l}\text { Avaliação de qualidade } \\
\text { de vida em crianças e } \\
\text { adolescentes asmáticos } \\
\text { usuários do Sistema } \\
\text { Único de Saúde (SUS) } \\
\text { em Recife-PE }\end{array}$ & ------ & $\begin{array}{l}\text { Aplicação do questionário pediat- } \\
\text { ric asthma quality of questionna- } \\
\text { rie - adaptado (PAQLQ-A) a crian- } \\
\text { ças, adolescentes e aos pais. }\end{array}$ \\
\hline Carmo (2007 & $\begin{array}{l}\text { Transtornos mentais } \\
\text { comuns em cuidadores } \\
\text { de crianças asmáticas: } \\
\text { um estudo transversal }\end{array}$ & ----- & $\begin{array}{l}\text { Realizou-se um estudo de corte } \\
\text { transversal. }\end{array}$ \\
\hline $\begin{array}{l}\text { Macedo et al. } \\
\text { (2007) }\end{array}$ & $\begin{array}{l}\text { Fatores de risco para } \\
\text { a asma em adultos, } \\
\text { Pelotas, Rio Grande do } \\
\text { Sul, Brasil }\end{array}$ & $\begin{array}{l}1968 \text { sujei- } \\
\quad \text { tos }\end{array}$ & $\begin{array}{l}\text { Um estudo populacional e trans- } \\
\text { versal, com aplicação de question- } \\
\text { ário. }\end{array}$ \\
\hline $\begin{array}{l}\text { Matos \& } \\
\text { Machado } \\
(2007)\end{array}$ & $\begin{array}{l}\text { Influência das variáveis } \\
\text { biopsicossociais na } \\
\text { qualidade de vida em } \\
\text { asmáticos }\end{array}$ & 50 sujeitos & $\begin{array}{l}\text { Para medir as variáveis sociode- } \\
\text { mográficas e clínicas, foi elaborada } \\
\text { e usada uma ficha de informação } \\
\text { clínica, recorrendo-se prioritari- } \\
\text { amente a entrevistas individual- } \\
\text { izadas com o doente e o médico } \\
\text { responsável pelo seu acompanha- } \\
\text { mento. }\end{array}$ \\
\hline
\end{tabular}




\begin{tabular}{|c|c|c|c|}
\hline Autor/ano & & Amostra & $\begin{array}{c}\text { Método } \\
\text { Instrumentos e delineamentos }\end{array}$ \\
\hline $\begin{array}{l}\text { Nogueira } \\
(2007)\end{array}$ & $\begin{array}{l}\text { Avaliação da qualidade } \\
\text { de vida em adolescen- } \\
\text { tes asmáticos }\end{array}$ & 210 sujeitos & $\begin{array}{l}\text { Estudo transversal de base am- } \\
\text { bulatorial. Para avaliação da QV, } \\
\text { usou-se um questionário auto- } \\
\text { preenchível, o paediatric asthma } \\
\text { quality of life questionnaire - } \\
\text { PAQLQ. }\end{array}$ \\
\hline $\begin{array}{l}\text { Peçanha \& } \\
\text { Lacharite } \\
(2007)\end{array}$ & $\begin{array}{l}\text { Avaliação familiar } \\
\text { sistêmica: sua validade } \\
\text { com crianças asmáticas } \\
\text { e suas famílias }\end{array}$ & 25 sujeitos & $\begin{array}{l}\text { Medidas incluíram o protocolo } \\
\text { AFS, o teste de desenho colorido } \\
\text { da família com as crianças (TDCF) } \\
\text { e uma entrevista semiestruturada } \\
\text { com os pais (EP). }\end{array}$ \\
\hline
\end{tabular}

Prevalência de trans-

Alvim et al. tornos emocionais e (2008) comportamentais em 464 sujeitos adolescentes com asma
Estudo transversal por meio de um questionário de transtornos psicológicos (strengths and difficulties questionnaire).
O impacto da asma,

Campanha, da rinite alérgica e

Freire \& Fon- da respiração oral na tes (2008) qualidade de vida de crianças e adolescentes
Levantamento bibliográfico.
Depressão e ansiedade

Carvalho (2008) em pacientes com asma e doença pulmonar obstrutiva crônica
Os dados foram comparados com 189 sujeitos grupo controle, levando-se em consideração aspectos demográficos, funcionais e de gravidade.

\begin{tabular}{|c|c|c|c|}
\hline $\begin{array}{l}\text { Matos \& } \\
\text { Machado } \\
(2008)\end{array}$ & $\begin{array}{l}\text { Estudo da psicoma- } \\
\text { nutenção da asma: } \\
\text { as cognições como } \\
\text { preditoras de emoções } \\
\text { e comportamentos } \\
\text { problemáticos associa- } \\
\text { dos à asma }\end{array}$ & 50 sujeitos & $\begin{array}{l}\text { Utilizou-se modelo cognitivo-com- } \\
\text { portamental, por meio de instru- } \\
\text { mentos de medida de variáveis } \\
\text { psicológicas (emoções e com- } \\
\text { portamentos) específicos para a } \\
\text { doença asmática. }\end{array}$ \\
\hline Nunes (2008) & $\begin{array}{l}\text { Intervenções fisioter- } \\
\text { apêuticas num con- } \\
\text { texto lúdico junto a } \\
\text { crianças com doenças } \\
\text { respiratórias crônicas }\end{array}$ & 58 sujeitos & $\begin{array}{l}\text { Empregou-se o método avaliativo- } \\
\text { interventivo quase-experimental. }\end{array}$ \\
\hline Ribeiro (2008) & $\begin{array}{l}\text { Asma e psicossomática } \\
\text { na infância: uma } \\
\text { análise de publicações } \\
\text { no periódico brasileiro } \\
\text { "Jornal de pediatria" }\end{array}$ & ------ & $\begin{array}{l}\text { Os artigos foram obtidos por meio } \\
\text { do site (www.sbp.com.br) da So- } \\
\text { ciedade Brasileira de Pediatria, no } \\
\text { link "Jornal de Pediatria". }\end{array}$ \\
\hline
\end{tabular}


Amostra

Método Instrumentos e delineamentos

Salomão Júnior et al. (2008)
Asma, competência social e transtornos comportamentais em crianças e adolescentes
Os pais das crianças responderam 124 sujeitos à versão brasileira do child behavior checklist.

$\begin{array}{ll}\text { Borba et al. } & \text { O mundo da criança } \\ \text { (2009) } & \text { portadora de asma } \\ & \text { grave na escola }\end{array}$

$\begin{array}{ll} & \begin{array}{l}\text { Violência no contexto } \\ \text { familiar enquanto um }\end{array} \\ \text { fator de risco para a } \\ \text { asma na infância }\end{array}$

Prevalência e fatores de risco para asma Jucá (2009) em escolares de 13 a 14 anos na cidade de Cuiabá-MT 1078 sujei- zando a conflict tactics scale - partos ent-child (CTSPC) e informações sobre a asma por meio do questionário ISAAC

Estudo transversal de base populacional com um estudo tipo casocontrole aninhado. Aplicação do questionário escrito modificado (QEM) do International Study of Asthma and Allergies in Childhood (ISAAC)

Estudo transversal com a metodologia do International Study of

3069 sujei- Asthma and Allergies in Childhood tos (ISAAC), utilizando-se questionário padronizado do ISAAC - Fase I e questionário complementar.

$\begin{array}{lll} & \text { Qualidade de vida } & \\ \text { Nogueira, } & \begin{array}{l}\text { em adolescentes } \\ \text { asmáticos: avaliação }\end{array} & \text { Utilizou-se questionário auto- } \\ \text { Silva \& Lopes } & \begin{array}{l}\text { 210 sujeitos } \\ \text { da gravidade da asma, }\end{array} & \begin{array}{l}\text { preenchível, o paediatric asthma } \\ \text { comorbidade e estilo }\end{array} \\ & \text { de vida } & \end{array}$

\section{Aspectos psicológicos como fatores desencadeantes da asma}

A asma constitui um risco em termos de adaptação e desenvolvimento psicológicos, principalmente ao que se refere a crianças e adolescentes. Segundo Salomão Júnior (2001), a presença de uma doença crônica na infância pode ser vista como um fator de impacto negativo sobre todo o processo de desenvolvimento, implicando restriçôes nas atividades habitualmente 
realizadas pela maioria das crianças (Rahim, 2000). O estudo realizado por L. M. Lima (2005) contribuiu para uma maior compreensão do impacto da asma na adaptação da criança. Procurou-se caracterizar sob um ponto de vista psicológico as crianças com asma e suas famílias, avaliando dimensões do temperamento, inteligência, autoestima, estratégias de coping, bem como a situação de estresse vivida no ano anterior ao estudo.

Os resultados encontrados por L. M. Lima (2005) demonstraram que crianças com asma apresentam dois perfis distintos de adaptação psicológica. $O$ perfil que indicou uma maior adaptabilidade era constituído por crianças que basicamente não apresentavam diferenças das crianças saudáveis, à exceção do coping. O estudo também apontou que as dimensões do temperamento, a eficácia percebida do coping e a autoestima constituem fatores preditores da adaptação psicológica da criança com asma.

Santos (2001) verificou, em seu trabalho, fatores que interferem no desenvolvimento e no agravamento de crises de asma, investigando aspectos emocionais envolvidos nessa patologia. Seus resultados permitem mostrar que as crises de asma estão intimamente vinculadas a fatores psicodinâmicos desenvolvidos em momentos específicos da vida de cada indivíduo e que, na amostra utilizada, os conflitos existenciais ocasionaram alta prevalência de ansiedade e dificuldade para enfrentar e ou resolver esse conflito.

Berenchtein (2004) investigou a influência do estresse na expressão clínica da asma e sua associação com as crises em crianças. Como resultado, observou-se que as crianças com asma estavam mais estressadas que as crianças do grupo controle, principalmente aquelas com maior gravidade da doença, o que pode indicar que a presença de estresse pode intensificar a frequência de sintomas da asma, a limitação à atividade física, o absenteísmo escolar e as interrupçôes do sono. O maior tempo de diagnóstico de asma implicou menor ocorrência de estresse, sugerindo a existência de um fator de adaptação à doença (Berenchtein, 2004). Com isso, pode-se inferir que o estresse é um fator importante no desencadeamento e agravamento das crises de asma nas crianças.

Os resultados encontrados nesses estudos dão indicativos de que a asma esteja vinculada a aspectos emocionais desenvolvidos em momentos específicos da vida do sujeito. Entre as variáveis mencionadas, o estresse foi citado como um fator importante no desencadeamento das crises, estando presente, principalmente, naqueles pacientes com maiores dificuldades de adaptação à doença. 
A asma, desse modo, foi revelada pelos dados das pesquisas como uma doença psicossomática, ou seja, um tipo de doença caracterizado por Mello Filho (2002) como qualquer doença do corpo, isto é, física, que se inicia ou se potencializa pela ação de fatores psicossociais no seu desencadeamento, evolução e agravamento.

\section{Aspectos psicológicos como fatores agravantes da doença}

Matos e Machado (2008) investigaram a associação entre as cogniçôes disfuncionais associadas à asma e outras variáveis psicológicas (emoções e comportamentos). Por cognições disfuncionais entendem-se distorções ou erros cognitivos relacionados às emoções e aos comportamentos, nesse estudo, ligados à asma. Os resultados encontrados sugerem que as cognições disfuncionais associadas à asma são preditoras de comportamentos/emoções problemáticos que ocorrem antes, durante e depois das crises asmáticas. Indicaram ainda que as cognições disfuncionais são preditoras de maiores níveis de sintomatologia "pânico/medo" e de atitudes negativas diante da doença asmática e ao seu tratamento (Matos \& Machado, 2008).

Peçanha (1997), em estudo sobre a reciprocidade de desenvolvimento entre a criança com asma e sua família, verificou que as crianças com asma apresentaram muitos problemas na área do comportamento e dificuldades emocionais para vivenciar determinadas situações em suas famílias, quando comparadas às crianças sem problemas de saúde. Os mesmos resultados foram encontrados por outros estudos (Resende, 2006; Santos, 2001), ao assinalarem que aspectos emocionais, físicos e ambientais se associam ao quadro da asma brônquica.

Existem evidências de que a asma está associada ao aumento da presença de sintomas psiquiátricos e de transtornos mentais. Partindo desse pressuposto, Valença et al. (2006) realizaram um estudo buscando averiguar a frequência de transtornos de ansiedade e depressão em uma amostra de pacientes asmáticos ambulatoriais e observaram se há relação desse tipo de comorbidade com a gravidade da asma. Apesar da alta frequência de depressão, 20 pacientes $(24,1 \%)$, e transtornos de ansiedade, 21 (33,8\%), apenas 4 (6,5\%) pacientes estavam em tratamento psiquiátrico e 13 (20,9\%) estavam usando benzodiazepínicos (Valença et al., 2006). Esses resultados sugerem a elevada comorbidade de transtornos de ansiedade e depressão em pacientes asmáticos, independente da gravidade da asma. 
O trabalho apresentado por Carvalho (2008) objetivou verificar a presença de sintomas de ansiedade e depressão entre os portadores de asma e doença pulmonar obstrutiva crônica. Para tanto, foram avaliados e quantificados os sintomas de ansiedade e depressão de 189 pacientes, sendo que a frequência de sintomas de ansiedade e depressão foi maior que entre aqueles com doença pulmonar obstrutiva crônica, o que pode dificultar o controle clínico. O estudo realizado por Alvim et al. (2008) apontou para o fato de que adolescentes com asma têm mais sintomas de transtornos emocionais e comportamentais do que os adolescentes em geral. $\mathrm{O}$ presente trabalho reforça a necessidade de uma abordagem sistêmica (biopsicossocial), multi e interdisciplinar do adolescente com asma.

Nota-se a concordância nos trabalhos aludidos à necessidade de uma abordagem sistêmica e interdisciplinar no que se refere à asma. Citando os transtornos emocionais e comportamentais como fatores agravantes da doença (Buzinari, 2001), salienta-se a importância de uma maior investigação nos possíveis aspectos psicológicos que estejam contribuindo na manutenção da asma. Os estudos consultados evidenciam uma associação entre aspectos psicológicos e a gravidade da asma, pois apontam que atitudes negativas diante da doença podem interferir no seu tratamento (Mendonça \& Ferreira, 2005). Dessa forma, esses trabalhos contribuem no sentido de incitar uma nova compreensão acerca da asma e suas causas, possibilitando o uso de intervenções adequadas às necessidades de cada paciente.

\section{Influência de fatores sociais ou psicossociais na asma}

Diante da etiologia diversa da asma infantil, os aspectos psicológicos vêm sendo reconhecidos como importantes para o desencadeamento e manutenção da doença. Como a criança é um ser em pleno processo de desenvolvimento físico e psíquico, além de suas próprias características, o papel da figura materna torna-se fundamental. Diferentes trabalhos (Leão, 2003; L. H. Lima, 2005; Oliveira, 2002; Rockenbach, 1993) apontam características comuns entre as crianças asmáticas, dificuldades das mães de relacionarem-se com seus filhos e a possibilidade de a dinâmica familiar perturbadora influenciar na asma infantil.

A asma é uma doença crônica mais comum na infância e tem um impacto psicossocial amplamente documentado, quer na criança, quer em sua família e em outros grupos de pertença. De tal modo, Rodrigues (1997) buscou avaliar características de um grupo de crianças asmáticas, suas respectivas mães e como estas vivenciam suas relações conjugais. Notou-se que as mães se perceberam 
em situações conflituosas, não satisfazendo eficazmente suas necessidades, com sentimentos de inferioridade e desproteção, com dificuldades nas relações interpessoais e conjugais. As crianças apresentaram conflitos ameaçadores e primitivos, atribuindo ao mundo externo a insatisfação de suas necessidades, com representações interiorizadas desfavoráveis das figuras parentais, autoimagem empobrecida e dificuldades de lidar com agressividade.

Entre as enfermidades crônicas infantis, a asma ganha destaque no âmbito familiar porque interfere na rotina da criança e dos pais (Peçanha \& Lacharite, 2007). As dificuldades emocionais dos pais impedem ajudar os filhos a amadurecerem, bloqueando tratamentos médicos e psicológicos (Fregonese, 2006). Considerando-se esses pressupostos, Fregonese pesquisou sobre o significado atribuído à maternidade e paternidade, além de investigar a associação entre o psicodinamismo de pais com a alergia respiratória do filho. Nesse estudo, as mães demonstraram a permanência de conflitos de uma forma imatura, pouco elaborados e demonstrando dificuldades em resolvê-los.

Carmo (2007) realizou um estudo visando a avaliar a influência de transtornos mentais maternos sobre a gravidade da asma infantil. Embora os valores encontrados não tenham sido estatisticamente significativos, encontrou-se que a condição de saúde mental materna está associada com a gravidade da asma, indicando que a presença de transtornos mentais comuns entre as mães contribuiu para a gravidade da doença.

O estudo realizado por Borba e Sarti (2005) apontou que a manifestação da asma mostra a necessidade de um tratamento interdisciplinar que contemple as dimensões biológica, psicológica e social na visão de uma assistência holista, que considere o paciente e sua família em seu mundo sociocultural. Dessa forma, os profissionais da saúde deverão contribuir para auxiliar a criança e a família a vivenciar a asma, como parte do processo dinâmico da vida.

A violência familiar contra a criança foi um tema recentemente inserido na literatura e tem se tornado relevante por ser um problema de saúde pública. Bonfim (2009) procurou estimar a associação entre a violência familiar perpetrada contra a criança e a ocorrência da asma. Idade materna jovem, suspeição de alcoolismo e baixa escolaridade foram características relacionadas a condições inadequadas de maternagem e têm sido associadas tanto à maior prevalência de asma quanto à violência familiar contra a criança.

De acordo com o que foi encontrado por L. H. Lima (2005), o momento da descoberta da doença é caracterizado por sentimentos de preocupação, dificuldade de lidar, mal-estar, tristeza, medo, susto e desespero. Para a mãe, 
cuidar de uma criança asmática significa preocupação, anulação, sofrimento, isolamento, limitação na vida da criança e ampliação das medidas de higiene ambiental. Pode-se concluir que a asma interfere de modo contundente na vida dessas mães e dos filhos.

O trabalho feito por Haidar (2004) teve como finalidade avaliar as características clínicas, socioeconômicas e laboratoriais dos pacientes asmáticos e averiguar a associação entre elas. Os principais fatores desencadeantes de crises foram as infecções de vias aéreas superiores, a poeira doméstica, as alterações climáticas e os exercícios físicos. As formas intermitentes e persistentes leves de asma ocorreram em $68,7 \%$ dos pacientes, e a rinite foi a doença alérgica mais frequentemente a elas associada, assim como no estudo realizado por Baldessar (2006) sobre os fatores de risco para a asma. Ribeiro, Furuyama, Schenkman e Jardim (2002) também encontraram resultados semelhantes, buscando uma associação entre infecções respiratórias e asma entre crianças mais novas.

Com o objetivo de avaliar a prevalência e fatores de risco para a asma na população adulta, Macedo et al. (2007) conduziram um estudo populacional e transversal em Pelotas, no Rio Grande do Sul. A prevalência de "sintomas atuais de asma" foi de $6 \%$. Os resultados salientaram a variação na prevalência de asma com diferentes critérios diagnósticos e que fatores genéticos, sociais e relacionados ao estilo de vida são relevantes na ocorrência da doença. A medida de qualidade de vida como um marcador de saúde vem sendo cada vez mais usada em vários estudos científicos no âmbito mundial, principalmente na avaliação de doenças crônicas (Aragão, 2007; Nogueira, 2007). Partindo de tal proposta, Nogueira, Silva e Lopes (2009) avaliaram a qualidade de vida de adolescentes asmáticos e sua associação com a gravidade da asma, doenças crônicas e estilo de vida. A qualidade de vida foi considerada ruim em metade dos adolescentes asmáticos de ambos os sexos. Dos meninos, $57 \%$ apresentavam qualidade de vida ruim, enquanto, nas mulheres, essa porcentagem era de $46 \%$, mas essa diferença não foi estatisticamente significativa. Esse estudo observou um declínio na qualidade de vida em todos os graus de gravidade de asma (leve, moderada e grave), sendo que houve uma maior associação entre asma grave e pior qualidade de vida.

A partir desses pressupostos, entende-se que fatores ambientais vêm sendo vinculados ao desencadeamento de crises em pacientes asmáticos. Essa inferência advém de dados que indicam fatores sociais implicados nesse processo, tais como uma relação familiar conturbada, disfunção na díade mãe-filho, questões emocionais dos pais e implicações no desempenho escolar, o que resulta, por sua vez, em prejuízos na qualidade de vida do 
asmático. Esses dados revelam esses fatores sociais como importantes meios de influência no surgimento e ou gravidade da asma.

\section{Influência de variáveis biopsicossociais em asmáticos}

A asma caracteriza-se como uma doença crônica que tem uma etiologia multifatorial, na qual fatores orgânicos e ou hereditários se combinam com fatores ambientais e psicológicos. Assim, a asma brônquica é uma doença complexa e interfere com a qualidade de vida desses doentes (Aragão, 2007; Breda, Freitas, Pizzichini, Agostinho \& Pizzichini, 2009). Nessa conjetura, Matos e Machado (2007) pretenderam estudar a relação das variáveis sociodemográficas (gênero, idade e grau de instrução), clínicas (gravidade da doença, duração e tipo clínico) e psicológicas (cognições, emoções e comportamentos) com a qualidade de vida do doente asmático. De um modo geral, os resultados dos estudos realizados (Matos \& Machado, 2007; Nunes, 2008; Ribeiro, 2008) confirmaram a associação entre as variáveis sociais, clínicas e psicológicas e os níveis de qualidade de vida dos doentes asmáticos, que se relacionam com um conjunto de variáveis que podem ser denominadas como biopsicossociais (idade, gênero, estatuto socioeconômico).

Os fatores determinantes do aparecimento de manifestações clínicas na asma constituem-se em fatores de risco. Estes são vários e podem ser denominados de maneiras diferentes: fatores predisponentes, que favorecem o aparecimento da doença; fatores causadores, que causam ou determinam o início da doença; fatores desencadeantes, que desencadeiam as exacerbações ou as crises da asma; e, ainda, os fatores agravantes, que podem piorar ou agravar os sintomas já presentes (Jucá, 2009; Lima, 2009). Alguns fatores, muitas vezes, podem atuar, tanto no desencadeamento como no agravamento dos sintomas.

Os dados apresentados pela pesquisa reforçam a importância de se desenvolverem programas de intervenção psicológica que visem a facilitar a adaptação da pessoa à realidade da doença, promovendo a sua qualidade de vida. De igual modo, sublinha a necessidade de se efetuar uma avaliação biopsicossocial, tendo em conta capacidades funcionais, aspectos psicológicos e o funcionamento social desses doentes, o que permitirá desenhar intervençôes que melhor se ajustem a cada doente e às suas necessidades.

Esses casos exemplificam com clareza um parâmetro de compreensão da asma brônquica: a correlação entre a dinâmica psicossomática e a periodicidade ou gravidade das crises, como ainda a relação entre dimensões 
psicológicas e sociais e a intensidade do sofrimento e a realidade do fenômeno orgânico da asma. Para repensar a asma na sua globalidade, torna-se necessário buscar uma concepção biopsicossocial. Essas dimensões interagem e estão permanentemente ligadas.

\section{Considerações finais}

Neste estudo, buscou-se levantar os principais achados acerca da relação entre asma e variáveis psicossociais e ou psicológicas, publicados em periódicos e banco de dados nacionais da Psicologia e ciências afins. Com o levantamento, constatou-se que, de um modo geral, os resultados confirmam a associação entre as variáveis psicológicas nos doentes asmáticos.

Com base nos resultados encontrados, evidencia-se que, ao avaliar quais variáveis influenciam a qualidade de vida relacionada com a asma, autores verificaram que a ansiedade constituía o seu principal preditor. Foi também demonstrado que dificuldades no âmbito das relações precoces com as figuras parentais são potencializadoras do desencadeamento da asma em crianças e que, para além de predispor e precipitar o desenvolvimento da asma, níveis elevados de estresse também se relacionam a uma pior qualidade de vida.

O ajustamento psicológico dos pais, em especial da mãe, é outro aspecto que apareceu como fator que influencia a resposta da criança à asma. Foi também demonstrado que a presença de sintomas depressivos na mãe está associada a uma série de crenças e atitudes que afetam de forma negativa o controle da asma dos seus filhos, nomeadamente a adesão ao tratamento e a utilização inadequada dos serviços de saúde.

As crianças e os adolescentes com asma apresentaram alteraçôes na competência social global e competência social relacionada a atividades cotidianas e escola. Apresentaram ainda transtornos globais e internalizantes, que podem prejudicar o seu desenvolvimento, sua qualidade de vida, a adesão ao tratamento e o manejo adequado da doença. Esses resultados sugerem que novas propostas terapêuticas englobem o envolvimento de equipes multiprofissionais, e os estudos reforçaram a necessidade de uma abordagem sistêmica do paciente asmático e seus familiares.

Pesquisas futuras, com amostras maiores e com diferentes grupos de crianças com doenças crônicas, poderiam auxiliar a compreender melhor o impacto psicossocial destas sobre o desenvolvimento. Além disso, estudos longitudinais são necessários para esclarecer as interações entre as variaçôes presentes no desenvolvimento normal e as variações decorrentes da presença 
da asma, tanto para a criança como para a família. Uma melhor compreensão da associação entre asma e variáveis psicológicas mostra-se fundamental para promover o desenvolvimento saudável dos asmáticos.

\section{Referências}

Alvim, C. G., Ricas, J., Camargos, P. A. M., Lasmar, L. M. B. L. F., Andrade, C. R. \& Ibiapina, C. C. (2008). Prevalência de transtornos emocionais e comportamentais em adolescentes com asma. Jornal Brasileiro de Pneumologia, 34 (4), 196-204.

Aragão, L. J. L. (2007). Avaliação de qualidade de vida em crianças e adolescentes asmáticos usuários do Sistema Único de Saúde (SUS) em Recife-PE. Dissertação de mestrado, Programa de Pós-graduação em Saúde da Criança e do Adolescente, Universidade Federal de Pernambuco, Recife.

Baldessar, M. Z. (2006). Fatores de risco para asma e rinite em adolescentes de criciúma. Dissertação de mestrado, Programa de Pós-graduação em Ciências da Saúde, Universidade do Extremo-Sul Catarinense, Criciúma.

Berenchtein, B. (2004). A influência do stress na expressão clínica da asma infantil. Dissertação de mestrado, Programa de Pós-graduação em Psicologia da Saúde, Universidade Metodista de São Paulo, São Paulo.

Bonfim, C. B. (2009). Violência no contexto familiar enquanto um fator de risco para sintomas asmáticos. Dissertação de mestrado, Programa de Pós-graduação em Saúde Coletiva, Universidade Federal da Bahia, Salvador.

Borba, R. I. H., Ribeiro, C. A., Ohara, C. V. S. \& Sarti, C. A. (2009). $\mathrm{O}$ mundo da criança portadora de asma grave na escola. Acta paulista de enfermagem, 22 (Especial - 70 anos), 921-927.

Borba, R. I. H. \& Sarti, C. A. (2005). A asma infantil e o mundo social e familiar da criança. Revista Brasileira de Alergia e Imunopatologia, 28 (5), 249254.

Breda, D., Freitas, F. F., Pizzichini, E., Agostinho, F. R. \& Pizzichini, M. M. M. (2009). Prevalência de sintomas de asma e fatores de risco associados em adolescentes escolares de 13 e 14 anos dos municípios de Tubarão e Capivari 
de Baixo, Santa Catarina, Brasil. Caderno de Saúde Pública, 25 (11), $2497-$ 2506.

Buzinari, M. (2001). Um estudo psicológico da asma em criança: consideraçôes acerca da angústia e inibição presente nessa sintomatologia. Dissertação de mestrado. Programa de Pós-graduação em Psicologia da Universidade Federal do Rio de Janeiro, Rio de Janeiro.

Campanha, S. M. A., Freire, L. M. S. \& Fontes, M. J. F. (2008). O impacto da asma, da rinite alérgica e da respiração oral na qualidade de vida de crianças e adolescentes. Revista Cefac, 10 (4), 513-519.

Carmo, M. B. B. (2007). Transtornos mentais comuns em cuidadores de crianças asmáticas: um estudo transversal. Dissertação de mestrado, Universidade Federal da Bahia, Salvador.

Carvalho, N. S. S. (2008). Depressão e ansiedade em pacientes com asma e doença pulmonar obstrutiva crônica. Dissertação de mestrado, Faculdade de Medicina, Universidade de São Paulo, São Paulo.

Castro, R. C. (2004). Alergias respiratórias e qualidade de vida: interface medicina e psicanálise. Dissertação de mestrado em Alergia e Imunopatologia, Universidade de São Paulo, São Paulo.

Fregonese, A. A. (2006). Estudo sobre o psicodinamismo de pais de crianças atendidas em programa de alegrias respiratórias. Dissertação de mestrado, Faculdade de Ciências Médicas da Santa Casa de São Paulo, São Paulo.

Haidar, D. M. C. (2004). Aspectos clínicos, socioeconômicos e laboratoriais de crianças asmáticas atendidas em um hospital público de São Luis-MA. Dissertação de mestrado, Programa de Pós-graduação em Ciências da Saúde, Universidade Federal do Maranhão, São Luís.

Jucá, S. C. B. M. P. (2009). Prevalência e fatores de risco para asma em escolares de 13 a 14 anos na cidade de Cuiabá-MT. Dissertação de mestrado, Programa de Pós-graduação em Saúde Coletiva, Universidade Federal de Mato Grosso, Cuiabá.

Leão, L. L. (2003). Depressão e transtornos psiquiátricos menores em mães de crianças com asma. Dissertação de mestrado, Universidade Católica de Pelotas, Pelotas.

Lemle, A. (2010). Aspectos psicossomáticos em pacientes com asma 
brônquica. In: J. de Mello-Filho (2010). Psicossomática Hoje. (2. ed.). Porto Alegre: Artmed.

Lima, L. M. M. (2005). A criança com asma: estudo de perfis de adaptação psicológica e de algumas variáveis preditivas. Dissertação de mestrado, Faculdade de Psicologia e de Ciências da Educação, Universidade de Porto, Porto.

Lima, L. H. O. (2005). Vivenciando o cuidar do filho asmático: respostas emocionais da mãe. Dissertação de mestrado, Programa de Pós-graduação em Enfermagem Universidade Federal do Ceará, Fortaleza.

Lima, W. L. (2009). Asma e fatores de risco associados em adolescentes de 13 e 14 anos de idade da cidade de São Luís, Maranhão. Dissertação de Mestrado em Saúde Materno-Infantil, Universidade Federal do Maranhão, São Luís.

Macedo, S. E. C., Menezes, A. M. B., Knorst, M., Dias-da-Costa, J. S., Gigante, D. P., Olinto, M. T. A. \& Fiss, E. (2007). Fatores de risco para a asma em adultos, Pelotas, Rio Grande do Sul, Brasil. Caderno de Saúde Pública, 23 (4), 863-874.

Matos, A. P. S. \& Machado, A. C. C. (2007). Influência das variáveis biopsicossociais na qualidade de vida em asmáticos. Psicologia: Teoria e Pesquisa, 23 (2), 139-148.

Matos, A. P. S. \& Machado, A. C. C. (2008). Estudo da psicomanutenção da asma: as cognições como preditoras de emoções e comportamentos problemáticos associados à asma. Psicologia USP, 19 (3), 393-413.

Mello Filho, J. (2002). Concepçãa psicossomática: visão atual. Rio de Janeiro: Tempo Brasileiro.

Mendonça, M. B. \& Ferreira, E. A. P. (2005). Adesão ao tratamento da asma na infância: dificuldades enfrentadas por cuidadoras. Revista brasileira de crescimento desenvolvimento humano, 15 (1), 56-68.

Nogueira, K. T. (2007). Avaliação da qualidade de vida em adolescentes asmáticos. Tese de doutorado, Instituto de Medicina Social, Universidade do Estado do Rio de Janeiro, Rio de Janeiro.

Nogueira, K. T., Silva, J. R. L. \& Lopes, C. S. (2009). Qualidade de vida em adolescentes asmáticos: avaliação da gravidade da asma, comorbidade e estilo de vida. Jornal de Pediatria, 85 (6), 523-530. 
Nunes, J. A. (2008). Intervençôes fisioterapêuticas num contexto lúdico junto a crianças com doenças respiratórias crônicas. Dissertação de mestrado, Universidade Metodista de São Paulo, São Paulo.

Oliveira, A. de. (2002). Função materna e os fenômenos psicossomáticos: reflexôes a partir da asma infantil. Dissertação de mestrado, Programa de Pós-graduação de Psicologia do Desenvolvimento, Universidade Federal do Rio Grande do Sul, Porto Alegre.

Peçanha, D. L. (1997). A reciprocidade de desenvolvimento entre a criança com asma e sua família. Tese de doutorado, Instituto de Psicologia, Universidade de São Paulo, São Paulo.

Peçanha, D. L. A. \& Lacharite, C. (2007). Avaliação familiar sistêmica: sua validade com crianças asmáticas e suas famílias. Psicologia em estudo, 12 (3), 503-512.

Rahim, M. A. A. (2000). Estudo da relação entre asma infantil e privação psicológica. Dissertação de mestrado, Programa de Pós-graduação em Psicologia Clínica, Instituto de Psicologia da Universidade de São Paulo, São Paulo.

Resende, V. D. G. (2006). Identificação dos fatores associados ao atraso do desenvolvimento motor em crianças de um a quatro anos de idade com asma. Dissertação de mestrado, Programa de Pós-graduação em Ciências da Reabilitação, Universidade Federal de Minas Gerais, Belo Horizonte.

Ribeiro, S. A., Furuyama, T., Schenkman, S. \& Jardim, J. R. B. (2002). Atopia, fumo passivo, infecções respiratórias e asma entre crianças do jardim de infância e da escola primária. São Paulo Medical Journal, 120 (4), 109-112.

Ribeiro, A. E. (2008). Asma e psicossomática na infância: uma análise de publicaçôes no periódico brasileiro "Jornal de pediatria". Dissertação de mestrado, Pontifícia Universidade Católica de São Paulo, São Paulo.

Rockenbach, D. L. P. (1993). Padrões de interação mãe-criança em díades com crianças asmáticas. Dissertação de mestrado, Programa de Pós-graduação em Psicologia do Desenvolvimento, Universidade Federal do Rio Grande do Sul, Porto Alegre.

Rodrigues, R. S. (1997). Contribuiçôes de técnicas de exame psicológico com crianças asmáticas e familiares. Dissertação de mestrado, Faculdade de Medicina de Ribeirão Preto, Universidade de São Paulo, Ribeirão Preto. 
Salomão Júnior, J. B. (2001). Competência Social e transtornos comportamentais em crianças portadores de asma moderada e grave. Dissertação de mestrado, Faculdade de Medicina de São José do Rio Preto, São José do Rio Preto.

Salomão Júnior, J. B., Miyazaki, M. C. O. S., Cordeiro, J. A., Domingos, N. A. M. \& Valério, N. I. (2008). Asma, competência social e transtornos comportamentais em crianças e adolescentes. Estudos em Psicologia, 25 (2), 185-192.

Santos, E. C. dos. (2001). Aspectos emocionais, fisicos e ambientais associados ao quadro de asma brônquica. Dissertação de mestrado, Programa de Pósgraduação de Psicologia da Saúde da Universidade Metodista de São Paulo, São Paulo.

Santos, R. C. A. N. (2004). Asma infantil: refletindo o cuidado materno. Dissertação de mestrado em Educação em Saúde, Centro de Ciências da Saúde, Universidade de Fortaleza, Ceará.

Valença, A. M., Falcão, R., Freire, R. C., Nascimento, I., Nascentes, R., Zin, W. A. \& Nardi, A. E. (2006). Relação entre a gravidade da asma e comorbidade com transtornos de ansiedade e depressão. Revista Brasileira de Psiquiatria, 28 (3), 206-208. 